ÉGYPTE monde arabe

\section{Égypte/Monde arabe}

$21 \mid 1995$

Économie égyptienne et perspectives de paix au Proche-Orient

\title{
Conséquences de la libéralisation du secteur agricole sur l'emploi
}

\section{Mûsa 'Abdal-'Azim}

\section{OpenEdition}

\section{Journals}

Édition électronique

URL : https://journals.openedition.org/ema/418

DOI : $10.4000 /$ ema.418

ISSN : 2090-7273

Éditeur

CEDEJ - Centre d'études et de documentation économiques juridiques et sociales

Édition imprimée

Date de publication : 31 mars 1995

Pagination : 188-195

ISSN : 1110-5097

Référence électronique

Mûsa 'Abdal-'Azim, «Conséquences de la libéralisation du secteur agricole sur l'emploi », Égypte/ Monde arabe [En ligne], 21 | 1995, mis en ligne le 08 juillet 2008, consulté le 07 juillet 2022. URL : http:// journals.openedition.org/ema/418; DOI : https://doi.org/10.4000/ema.418

Ce document a été généré automatiquement le 7 juillet 2022

Tous droits réservés 


\title{
Conséquences de la libéralisation du secteur agricole sur l'emploi
}

\author{
Mûsa 'Abdal-'Azim
}

1 Fondée sur la libéralisation de la production, du commerce, des prix et des baux locatifs ainsi que sur la privatisation, la réforme du secteur agricole égyptien a débuté à la fin des années 80 et se poursuit depuis 1990 avec le programme de stabilisation et d'ajustement structurel. Pour analyser ses effets quantitatifs et qualitatifs sur l'emploi agricole, nous aborderons successivement les caractéristiques de la population active agricole, les salaires, l'impact de l'exode rural et de l'émigration, celui de la mécanisation et enfin, l'offre et la demande de main-d'oeuvre agricole. Caractéristiques de la population active agricole. La population égyptienne a augmenté de 30 à 48 millions d'habitants entre 1966 et 1986, et sa population active employée ${ }^{1}$ âgée de plus de 6 ans, de 7,6 à 13,4 millions entre ces deux dates, avec près de 300.000 actifs supplémentaires par an. Dans le même temps, la population active agricole ${ }^{2}$ employée est passée de 3,7 à 4,6 millions, soit une augmentation annuelle moyenne de 45.000 actifs agricoles ${ }^{3}$.

2 La part de l'agriculture dans l'emploi total n'a cessé de diminuer depuis 30 ans; selon les recensements, elle a baissé de $51 \%$ en 1966 à $43 \%$ en 1976, puis à $36 \%$ en $1986^{4}$. Avec 8 millions d'actifs en 1988, l'agriculture reste néanmoins le premier secteur d'emploi en Égypte. Les moins de 12 ans et les plus de 64 ans, ainsi que les femmes, y sont relativement plus nombreux que dans les autres branches ${ }^{5}$. Ces trois catégories représentent respectivement $6 \%, 4 \%$ et $51 \%$ de l'emploi agricole, alors qu'elle ne constituent que $3 \%, 3 \%$ et $35 \%$ de la population active totale. L'analphabétisme est plus répandu chez les agriculteurs (76\%) que chez les autres actifs (51\%) et davantage chez les agricultrices (90\%) que chez les agriculteurs (63\%). Les activités du secteur agricole égyptien sont peu diversifiées, puisque $97 \%$ de ses actifs travaillent la terre, les $3 \%$ restants occupant des fonctions administratives, techniques ou commerciales. Les travailleurs familiaux non rémunérés, composés essentiellement des femmes, des enfants et des personnes âgées, y sont majoritaires (58\%), suivis des chefs d'exploitation (23\%) et des salariés (17\%), parmi lesquels les hommes dominent (80\%). 
L'emploi saisonnier ou intermittent ne concerne que $18 \%$ des actifs agricoles, essentiellement masculins (76\%).

Les salaires

3 Dans l'agriculture des pays en développement, la détermination des salaires ne vérifie pas la théorie économique, qui affirme que le salaire doit être égal au produit marginal du travail. D'une part, les salariés sont le plus souvent des saisonniers ou des travailleurs à la tâche. De plus, les salaires, monétaires ou en nature, y sont plus bas que dans l'industrie ou les services, bien que les journées de travail y soient plus longues. D'après une étude sur échantillon réalisée en $1984 / 85^{6}$, les salaires quotidiens moyens agricoles des trois groupes d'actifs (hommes, femmes et enfants) atteignaient respectivement 5,5 livres égyptiennes (LE), 3,7 LE et 2,6 LE, tandis que le salaire journalier moyen non agricole s'élevait à 7,3 LE pour les hommes et 5,3 LE pour les femmes. Le salaire moyen agricole réel a augmenté à partir de 1974 et jusqu'en 1986. Le salaire journalier réel moyen est ainsi passé, aux prix constants 1974, de 0,35 LE en 1974 à $1 \mathrm{LE}$ en 1986. A partir de cette date, le salaire réel diminue pour atteindre 0,62 LE en 1989 , suite à ${ }^{7}$ la libéralisation du commerce et des prix des intrants, des produits et des services ainsi que la suppression des subventions aux produits intermédiaires et finis ${ }^{8}$. Impact de l'exode rural et de l'émigration

Depuis trente ans, l'exode rural a fortement ralenti grâce à l'amélioration des services ruraux et à la possibilité de construire des logements à des coûts relativement bas sur les terrains agricoles. Mais l'émigration vers les pays arabes pétroliers, dont le volume aurait atteint 2 millions de personnes et $42 \%$ de ruraux en $1987^{9}$, a réduit et féminisé l'offre de main-d'œuvre agricole, tout en favorisant la hausse des salaires. Depuis la fin des années 80, et surtout après la seconde guerre du Golfe en 1990, l'émigration a fortement diminué, les salaires nominaux stagnent et les salaires réels agricoles ont sensiblement baissé.

Impact de la mécanisation

5 Les années 70 et 80 ont vu les outils traditionnels tels que l'araire (mihrâth balâdî), les élévateurs d'eau par balancier (chadû) ou par une roue mue par du bétail (sâqiyya) ainsi que des animaux de trait, remplacés par les pompes à moteur, les tracteurs et les moissonneuses-batteuses; on recense cinq fois plus de chevaux moteurs qu'il y a 20 ans, passés de 1.201 à 6.867 entre 1970 et 1989. Le nombre d'hommes/jours travaillé a augmenté en 1989 par rapport à 1970 pour certaines cultures comme le trèfle d'Alexandrie (bersîm), la canne à sucre et le coton. Il a faiblement diminué pour les fruits, les légumes, le blé, les fèves, le riz d'été et le mais (dhura châmiyya), grâce à l'utilisation du sarcloir ('azaqaf) pour les fruits et légumes, et à celle des moissonneuses dans la culture du blé et du riz. Toutefois, selon l'étude de Abû-l-Wafâ (1988) sur les périmètres cultivés, la mécanisation a peu réduit l'emploi agricole, excepté pendant la récolte du riz, grand consommateur de travail mécanisé. En effet, les paysans des anciennes terres de la Vallée, qui constituent l'essentiel de la superficie cultivée en Égypte, utilisent encore peu de machines et seulement à quelques tâches ponctuelles. Les gains de productivité liés à la mécanisation sont faibles. Le travail mécanisé coûte plus cher que le travail simple qu'il remplace, et les personnels qualifiés comme les conducteurs d'engins, les mécaniciens et les électriciens manquent, ainsi que les pièces de rechange. La mécanisation est plus développée sur les terres nouvellement conquises sur le désert. Les techniques y sont plus avancées et la main-d'œuvre peu nombreuse, et elles peuvent absorber davantage de travail mécanique et animal. D'où 
l'importance de la formation et de l'enseignement technique agricole. Les hausses de prix, la stagnation des salaires et l'expansion du chômage dans les années 90, pourraient accroître le coût relatif de la mécanisation et en ralentir la progression.

offre et demande de main-d'œuvre agricole présente et future.

6 Selon l'enquête par échantillon de 1988, le travail agricole annuel s'élève à 106 millions de journées ouvrées. Ce chiffre global de l'emploi reflète des situations très différentes, selon le statut du travailleur (propriétaire, fermier ou ouvrier), son sexe, la taille de l'exploitation, la période de l'année, le type de culture, la zone géographique, etc. L'enquête par échantillon évalue l'emploi agricole annuel moyen par sexe à 280 jours/ homme, 188 jours/femme et 159 jours/enfant, fonctionnaires et employés du secteur public non compris. Dans les petites exploitations familiales, qui prédominent en Égypte, de nombreux facteurs limitent les besoins en main d'œuvre: la dispersion des parcelles, le manque de coopération et de planification entre exploitants, le manque de terre et capital, l'importance de l'autoconsommation relativement à la production marchande. Dans les exploitations de plus de cinq feddans, employant moins de quatre membres de la famille, l'emploi varie davantage, selon le calendrier. L'ouvrier agricole, qui ne travaille en moyenne que le tiers de l'année, est, outre le chômage saisonnier, en situation constante de sous-emploi. Le chômage traditionnel agricole commun aux ouvriers qualifiés et non qualifiés s'élèverait à 1,4 million de personnes, particulièrement les femmes rurales, en supposant que $20 \%$ d'entre elles ne souhaitent pas travailler.

7 Le développement de l'emploi agricole est un véritable défi pour la réforme économique. Selon le Bureau international du travail, la population active augmentera chaque année, de 2,5\%, soit 4,54 millions de travailleurs supplémentaires d'ici 2000. En tenant compte des 600.000 chômeurs de 1990, il faudrait créer 650.000 emplois nouveaux par an pour occuper les 6,5 millions de demandeurs d'emploi qui apparaîtront au cours des années 90 , sans compter la baisse de la demande de salariés des micro-exploitations familiales.

Même si le chômage apparent est source de problèmes sociaux et économiques, le développement de l'emploi ne peut être envisagé que s'il correspond à de véritables postes de travail. La politique de libéralisation, outre les réformes monétaires, financières et institutionnelles indispensables à l'assainissement de l'économie nationale, doit résorber le chômage en promouvant des techniques intensives en travail. Elle doit inciter les banques et le Fonds social à aider les petits établissements à investir, favoriser des emplois d'utilité publique dans les ONG, les syndicats et les coopératives, aider à créer des entreprises de services agricoles et à installer des jeunes sur des terres nouvelles, favoriser la durée d'exploitation des machines par généralisation du travail en équipes. Ces mesures devraient permettre d'absorber une grande part de l'excédent de main-d'œuvre prévu pour la décennie à venir.

Traduit de l'arabe par Samia Rizq. 


\section{BIBLIOGRAPHIE}

AL-ISAWI l., "Y a-t-il un chômage déguisé dans l'agriculture ? ", Misr al-Qâhira n 270, octobre 1977.

GUWAYLI A. A. \& IBRAH1M A. Y, « Le rôle de la femme dans la production agricole », Colloque national sur Le rôle de la femme dans l'agriculture et la sécurité alimentaire, FAO, Le Caire, 1984.

ACHARI A. M., « Evaluation de la demande de travail agricole pour certaines cultures du gouvernorat d'al-Gharbiyya ", IX Congrès international de la statistique, de la comptabilité scientifique, des études sociales et de la population, 1984.

ABD AL-RAZIQ RADI A., «L'émigration de la main-d'œuvre égyptienne vers le Golfe », Union des associations de développement administratif et Agence centrale d'organisation et d'administration. Colloque sur L'émigration de la main-d'œuvre égyptienne, Janvier 1984.

TUBAR S., «Le programme de réforme économique et la question du chômage ", Congrès sur La stratégie de l'agriculture égyptienne dans les années 90, Ministère de l'Agriculture et de la bonification des terres, secteur des affaires économiques, 1992.

\section{ANNEXES}

Tableau 1 : Population active totale et pop. agricole par zone et par sexe (en milliers)

\begin{tabular}{|l||l|l|l|l|l|l|}
\hline \multicolumn{1}{|l||}{} & \multicolumn{2}{|l|}{ Zones rurales } & \multicolumn{2}{l|}{ Zones urbaines } & \multicolumn{2}{l|}{ Total } \\
\cline { 2 - 7 } & Hommes & Femmes & Hommes & Femmes & Hommes & Femmes \\
\hline \hline Agriculture & 3.696 & 3.762 & 229. & 339 & 3.925 & 4.101 \\
\hline \hline Total & 6.415 & 4.497 & 4.745 & 1.607 & 11.160 & 6.104 \\
\hline \hline \% agriculture & $58 \%$ & $84 \%$ & $5 \%$ & $21 \%$ & $35 \%$ & $57 \%$ \\
\hline
\end{tabular}

Source : CAPMAS. enquête main-d'œuvre par échantillon 1988.

Tableau 2 : Besoins en main-d'œuvre des principales cultures (Jour/homme)

\begin{tabular}{|l|l|l|l|l|l|l|}
\hline & 1970 & 1980 & 1989 & 1992 & $1970-79$ & $1980-89$ \\
\hline \hline Blé & 27,02 & 26,52 & 24,72 & 23,9 & $-2 \%$ & $-7 \%$ \\
\hline \hline Fève & 14,18 & 14,90 & 12,11 & 13.9 & $5 \%$ & $-19 \%$ \\
\hline \hline Trèfle & 23,73 & 22,80 & 25,12 & 25,9 & $-4 \%$ & $10 \%$ \\
\hline
\end{tabular}




\begin{tabular}{|l|l|l|l|l|l||l|}
\hline Coton & 83,96 & 84,93 & 84,96 & 85,0 & $1 \%$ & $0 \%$ \\
\hline \hline Canne 1ère & 137,93 & 131,00 & 144,37 & 172,4 & $-5 \%$ & $10 \%$ \\
\hline \hline Canne 2e & 63,49 & 62,87 & 59,05 & 58,2 & $-1 \%$ & $-6 \%$ \\
\hline \hline Riz été & 55,50 & 53,92 & 52,01 & 52,0 & $-3 \%$ & $-4 \%$ \\
\hline \hline Maïs été & 31,70 & 32,24 & 30,39 & 30,4 & $2 \%$ & $-6 \%$ \\
\hline \hline Sorgho été & 47,48 & 54,83 & 54,93 & 43,0 & $15 \%$ & $0 \%$ \\
\hline \hline Légumes & 57,75 & 54,62 & 48,43 & 48,4 & $-5 \%$ & $-11 \%$ \\
\hline \hline Fruits & 124,06 & 120,65 & 113,31 & 116,7 & $-3 \%$ & $-6 \%$ \\
\hline \hline Total & $2.636,8$ & $2.639,28$ & $2.638,40$ & $2.651,0$ & $0 \%$ & $0 \%$ \\
\hline
\end{tabular}

Source : calculé à partir des données du Centre d'études agricoles (non publiées).

\section{NOTES}

1. Toute personne exerçant normalement une activité permanente mais ne travaillant pas au cours de la semaine pendant laquelle est effectuée l'enquête main-d'œuvre par échantillon ou le recensement, est considérée comme « active employée » sauf si elle se trouve en congé sans solde depuis au moins un an. Le chômeur est défini comme appartenant à la population active, désireux de travailler et prêt à le faire mais n'exerçant aucune activité économique au cours de la semaine de l'enquête ou du recensement.

2. Sont inclus dans la population active agricole les actifs travaillant dans l'agriculture en zone rurale ou urbaine.

3. CAPMAS, Livre statistique annuel de la RAE, 1952-90, édition 1991, et Enquête main-d'œuvre par échantillon, 4e trimestre 1988, Le Caire, avril 1991. 4. L'Enquête main-d'œuvre par échantillon d'octobre 1988 prend en compte les fonctionnaires, les employés du secteur public ainsi que les employés des professions techniques, scientifiques, administratives et les employés de bureau, non compris dans les résultats du recensement publiés par le Livre statistique annuel jusqu'à l'édition de 1991. Pour cette raison, les résultats de l'enquête par échantillon d'octobre 1988 ne peuvent être comparés à ceux des enquêtes précédentes ou à ceux du recensement. Par ailleurs, le recensement général de la population donne une estimation beaucoup plus faible de l'emploi en général et de l'emploi agricole en particulier que l'Enquête main-d'œuvre par échantillon. Selon le premier, la population active ne comptait que 13,4 millions de personnes en 1986 alors que d'après la seconde, elle en comptait déjà 17,2 millions en 1988. Selon cette même enquête, l'agriculture occuperait encore $47 \%$ de la population active totale en 1988. L'important écart entre les 
deux sources n'infirme pas la tendance longue à la réduction de la part de l'agriculture dans l'emploi confirmée par les deux sources statistiques (NDLR). 5. Celle proportion d'enfants et de personnes âgées actives plus élevée dans l'agriculture que dans les autres secteurs s'explique par l'existence de tâches simples nécessitant un faible niveau technique ou peu de force physique. Les plus âgés prennent en charge la gestion de l'exploitation ou sa surveillance, les plus jeunes gardent le bétail, désherbent ou guident les ânes transportant le fumier vers les champs, tandis que les femmes effectuent des travaux simples (confection des fromages et du beurre, élevage de la volaille) ou aident les hommes pendant la récolte, en plus de leurs tâches ménagères et de l'éducation des enfants.

6. Abu-l-Wafa' A. L. et alii, Analyse économique et sociale de la main-d'œuvre agricole et de ses problèmes en RAE, Académie de la recherche scientifique et technologique, Institut d'études de l'alimentation et de l'agriculture, branche d'économie agricole et de développement social, Alexandrie. 1988.

7. L'indice des prix à la consommation passe de $16,7 \%$ en $1985 / 86$ à $22,2 \%$ en 1989/90. Abu Mandur Al-Dib M., La population active agricole égyptienne : transformations des décennies 70 et 80 et prospective des années 90 , Colloque national des politiques agricoles en RAE, FAO, 1992.

8. Voir indice des prix à la consommation en annexe du colloque (NDLR).

9. Enquête sur l'émigration du CAPMAS 1987 (NDLR).

INDEX

Mots-clés : agriculture, libéralisation 